\title{
Measuring the Similarity between zSlices General Type-2 Fuzzy Sets with Non-Normal Secondary Membership Functions
}

\author{
Josie McCulloch and Christian Wagner \\ Lab for Uncertainty in Data and Decision Making (LUCID) and Horizon, School of Computer Science, \\ University of Nottingham, Nottingham, NG8 1BB, UK \\ Email: \{josie.mcculloch; christian.wagner\}@nottingham.ac.uk
}

\begin{abstract}
This paper presents a method of measuring the similarity between general type-2 fuzzy sets that may have nonnormal secondary membership functions. Such fuzzy sets are increasingly common in applications such as the modelling of the subjective meaning of linguistic terms by groups of people. By building upon existing similarity measures in the literature, which thus far cannot compare such fuzzy sets, we derive an extended similarity measure which can be applied to both normal and nonnormal (in terms of the secondary membership functions) general type-2 fuzzy sets. We provide proofs that the proposed method follows all of the common properties of a similarity measure and demonstrations are given to compare the proposed method with others in the literature.
\end{abstract}

Index Terms-similarity measure, fuzzy sets, general type-2, zSlices, $\alpha$-planes

\section{INTRODUCTION}

The concept of similarity is used to recognise patterns and associations between objects and concepts. Determining the similarity between two objects is important in many applications, both inside and outside of the field of fuzzy set theory. Due to the complex and context-dependent nature of defining similarity, there have been many measures developed to compare type-1 [1]-[3], interval type-2 [4], [5], and general type-2 [6]-[10] fuzzy sets. These methods have been used in a variety of applications, such as computing with words [4], [11], data mining [12] and clustering [13], [14].

Recently, the zSlices (a.k.a. $\alpha$-plane) representations of general type-2 fuzzy sets have gained some attention [15][19] and methods of measuring the similarity using this representation have been developed [6]-[8]. However, such current measures assume that the fuzzy sets have normal secondary membership functions. As a result, they cannot be used to calculate the similarity between fuzzy sets with nonnormal secondary membership functions. Such fuzzy sets may arise, for example, if there is no consensus when modelling agreement between individuals or, in some contexts, it may not make sense to describe a term as ever being completely certain [20]. This paper presents a method of measuring the similarity between such fuzzy sets.

This work was partially funded by the EPSRC's Towards Data-Driven Environmental Policy Design grant, EP/K012479/1, RCUK's EP/K014234/2 Prototyping Open Innovation Models for ICT-Enabled Manufacturing in Food and Packaging, and EP/M02315X/1 From Human Data to Personal Experience.
The proposed approach is based on current techniques in the literature and produces consistent results when compared with existing methods. This method follows all of the properties commonly desired in similarity measures and may be used to compare fuzzy sets with both normal or non-normal secondary membership functions.

The remainder of this paper is structured as follows. Section II presents the reader with the necessary background on fuzzy sets and similarity measures. After this, Section III presents a new similarity measure that can be used to compare zSlices-based fuzzy sets that may have non-normal secondary membership functions. Demonstrations of this new measure are shown in Section IV, and conclusions are presented in Section V.

\section{BACKGROUND}

This section first presents background on fuzzy sets followed by an overview of similarity measures on zSlices-based fuzzy sets within the literature.

\section{A. Fuzzy Sets}

The concept of a fuzzy set is a useful model for representing uncertainty. Unlike crisp set theory, in which an element or object completely belongs or does not belong to a set, in fuzzy set theory an element can be described as partially belonging to a fuzzy set. This uncertainty is represented within the interval $[0,1]$ where 0 and 1 indicate no membership and full membership, respectively.

1) Type-1 Fuzzy Sets: Let $T 1(X)$ represent the set of all type- 1 fuzzy sets in the universe of discourse $X$.

Definition 1. A fuzzy set $A \in T 1(X)$ may be represented as a set of ordered pairs

$$
A=\left\{\left(x, \mu_{A}(x)\right) \mid x \in X\right\},
$$

where $\mu_{A}(x)$ is the membership value of the element $x$ within $A$ and $\mu_{A}(x) \in[0,1]$.

(1) is also sometimes expressed as

$$
A=\int_{x \in X} \mu_{A}(x) / x,
$$


where $\int$ does not indicate integration but instead denotes the collection of all points $x$ in $X$ with associated membership function $\mu_{A}(x)$.

Definition 2. A fuzzy set $A \in T 1(X)$ may be described as normal or non-normal. $A$ is normal if its height $h_{y}(A)$ (in which $y$ refers to the height on the $y$, a.k.a. $\mu$, axis), defined as $\max _{x \in X} \mu_{A}(x)$, equals 1.0; i.e. $\exists x \in X, \mu_{A}(x)=1$.0. If $h_{y}(A)<1.0$ then $A$ is said to be non-normal.

Fuzzy sets are defined by their membership functions. The most common shapes of membership functions are triangular, trapezoidal and Gaussian. This paper will provide examples based on fuzzy sets with triangular membership functions, which for $A \in T 1(X)$ is defined as $\mu_{A}=\operatorname{trimf}(x ;[a, b, c ; w])$ such that the membership value of any given $x \in X$ is

$$
\mu_{A}(x)= \begin{cases}w(x-a) /(b-a) & a \leq x \leq b \\ w(c-x) /(c-b) & b \leq x \leq c \\ 0 & \text { otherwise }\end{cases}
$$

where $w$ is a weight used to adjust the height of the membership function. Note that $h_{y}(A)=w$. In Section IV, demonstrations of similarity measures will be given using fuzzy sets with triangular membership functions. Note, however, that the measures demonstrated in this paper may be used on fuzzy sets with membership functions of any shape.

2) General Type-2 Fuzzy Sets: In a type-1 fuzzy set $A$, the degree of membership of $x$ in $A$ is a value within $[0,1]$. However, type-2 fuzzy sets model the membership of $x$ as a type-1 fuzzy set (which has a universe of discourse within $[0,1])$. This is referred to as a secondary membership function and it enables one to express uncertainty in the membership of $x$. Let $G T 2(X)$ represent the set of all general type-2 fuzzy sets in $X$.

Definition 3. A fuzzy set $\tilde{A} \in G T 2(X)$ is expressed as [21]

$$
\tilde{A}=\left\{\left((x, u), \mu_{\tilde{A}}(x, u)\right) \mid \forall x \in X, \forall u \in J_{x} \subseteq[0,1]\right\},
$$

where $x$ is the primary variable in $X, u$ is the secondary variable which has the domain $J_{x} \subseteq[0,1]$, and the amplitude of $\mu_{\tilde{A}}(x, u)$ is known as the secondary grade.

Many different representations of general type-2 fuzzy sets have been developed in the literature. The most well known method of defining a general type-2 fuzzy set is as a collection of vertical slices; this is the method shown in (4). Another common representation is the zSlices approach, which will be the focus of this paper. zSlices general type-2 fuzzy sets are based on the theory of interval type- 2 fuzzy sets, and so the next section introduces the latter followed by a section discussing the zSlices approach.

3) Interval Type-2 Fuzzy Sets: An interval type-2 fuzzy set is equivalent to a general type-2 fuzzy set in which all secondary membership values are 1 ; i.e. $\mu(x, u)=1, \forall x \in$ $X, \forall u \in J_{x} \subseteq[0,1]$. Let $I T 2(X)$ represent the set of all interval type-2 fuzzy sets within $X$.
Definition 4. The fuzzy set $\tilde{A} \in I T 2(X)$ is written as [22]

$$
\tilde{A}=\left\{\left((x, u), \mu_{\tilde{A}}(x, u)=1\right) \mid \forall x \in X, \forall u \in J_{x} \subseteq[0,1]\right\} .
$$

Note, this is a vertical slice representation where $J_{x}$ denotes each vertical slice.

Definition 5. The vertical slice $J_{x}$ of $\tilde{A} \in I T 2(X)$ is written as [22]

$$
J_{x}=\left[\underline{\mu}_{\tilde{A}}(x), \bar{\mu}_{\tilde{A}}(x)\right], \forall x \in X,
$$

where $\underline{\mu}_{\tilde{A}}(x)$ and $\bar{\mu}_{\tilde{A}}(x)$ refer to the lower and upper membership functions, respectively. The bounded region $J_{x}$ is commonly referred to as the footprint of uncertainty; this is the region where $\mu(x, u)=1$.

4) The zSlices/alpha-Plane Approach: This paper focuses on the zSlices approach [23] and $\alpha$-plane model [24], which both involve slicing a fuzzy set along the secondary membership axis. Although these two methods go by different names, the theory is equivalent [25]. This approach represents a general type-2 fuzzy set as a collection of interval type-2 fuzzy sets with a third dimension that may be different to 1 . For continuity, only the zSlices notations will be used throughout this paper.

A zSlices type-2 fuzzy set can be composed by slicing a general type- 2 fuzzy set along the $z$-axis (or the $\mu(x, u)$ axis). This breaks the fuzzy set down into many interval type-2 fuzzy sets called zSlices. However, unlike regular interval type-2 fuzzy sets that have a secondary membership grade of 1 , each zSlice has a height of $z_{i}$, referred to as the zLevel.

Definition 6. Given a general type-2 fuzzy set $\tilde{A}$, let the zSlice of $\tilde{A}$ at the level $z_{i}$ be denoted $\tilde{A}_{z_{i}}$. This is written as [23]

$$
\tilde{A}_{z_{i}}=\left\{\left((x, u), \mu_{\tilde{A}_{z_{i}}}(x, u)=z_{i}\right) \mid \forall x \in X, \forall u \in J_{x} \subseteq[0,1]\right\} .
$$

(7) is the vertical slice representation of an individual zSlice. This is akin to the interval type-2 representation (5) but the secondary membership value is always $z_{i}$. Note that, throughout this paper, $x, y$ and $z$ correspond to the universe of discourse, primary membership and secondary membership (zLevel), respectively.

Definition 7. The fuzzy set $\tilde{A} \in G T 2(X)$ is represented as a collection of its zSlices [23] as

$$
\tilde{A}=\sum_{i=1}^{I} \tilde{A}_{z_{i}}
$$

where $\sum$ denotes the union of zSlices $\tilde{A}_{z_{i}}$ and $I$ is the total number of zSlices.

Note that the zSlice $Z_{0}$ is disregarded in (8) because its secondary grade is 0 and thus it does not contribute to the fuzzy set [23].

Definition 8. Let zIT2 denote the set of all zSlices which are equivalent to an interval type-2 fuzzy set but with secondary membership values at a known value $z_{i}$. 
Definition 9. Let $\tilde{A}_{Z}$ denote the set of all zLevels of the zSlices in $\tilde{A}$. This is defined as

$$
\tilde{A}_{Z}=\left\{z_{i} \mid \tilde{A}_{z_{i}} \neq \emptyset, \forall i \in\{1,2, \ldots, I\}\right\},
$$

where $I$ is the total number of zLevels in $\tilde{A}$.

Definition 10. Let $h_{z}(\tilde{A})$ denote the maximum height over all zSlices in $\tilde{A} \in G T 2(X)$. This is defined as the maximum zLevel in (9); i.e.,

$$
h_{z}(\tilde{A})=\max \left\{z_{i} \mid \tilde{A}_{z_{i}} \neq \emptyset, \forall i \in\{1,2, \ldots, I\}\right\} .
$$

If the value of (10) is less than 1.0 then $\tilde{A}$ has a non-normal secondary height.

Having given an overview of fuzzy sets, the next section presents methods of calculating the similarity between fuzzy sets within the literature.

\section{B. Similarity Measures}

A similarity measure on fuzzy sets is a function $s: A \times B \rightarrow$ $[0,1]$ that determines to what degree two type- 1 or type-2 fuzzy sets $A$ and $B$ contain the same values with the same degree of membership. The most common properties of a similarity measure include:

Reflexivity: $s(A, B)=1 \Longleftrightarrow A=B$

Symmetry: $s(A, B)=s(B, A)$

Overlapping: If $A \cap B \neq \emptyset$, then $s(A, B)>0$; otherwise, $s(A, B)=0$

Transitivity: If $A \subseteq B \subseteq C$, then $s(A, B) \geq s(A, C)$ Regarding transitivity, note the following definitions:

Definition 11. For $A, B \in T 1(X), A \subseteq B$ if $\mu_{A}(x) \leq \mu_{B}(x)$, $\forall x \in X[26]$.

Definition 12. For $\tilde{A}, \tilde{B} \in I T 2(X), \tilde{A} \subseteq \tilde{B}$ if $\underline{\mu}_{\tilde{A}}(x) \leq$ $\underline{\mu}_{\tilde{B}}(x)$ and $\bar{\mu}_{\tilde{A}}(x) \leq \bar{\mu}_{\tilde{B}}(x), \forall x \in X$ [27].

Definition 13. For $\tilde{A}, \tilde{B} \in G T 2(X), \tilde{A} \subseteq \tilde{B}$ if $\tilde{A}_{z_{i}} \subseteq$ $\tilde{B}_{z_{i}}, \forall i \in\{1,2, \ldots, I\}$ where $I$ is the total number of zSlices in $\tilde{A}$ and $\tilde{B}$ and the subsethood of $\tilde{A}_{z_{i}}, \tilde{B}_{z_{i}} \in z I T 2(X)$ is the same as given in Definition 12 [8].

Note that it is not necessary for a similarity measure to have all four properties as the desired properties of the measure depend on its application [28]-[30].

One of the most common similarity measures on fuzzy sets is the Jaccard measure. Let $\mathscr{P}(\mathbb{R})$ be the set of all crisp sets in $\mathbb{R}$, then for two groups $U, V \in \mathscr{P}(\mathbb{R})$ the Jaccard similarity is [31]

$$
s(U, V)=\frac{|U \cap V|}{|U \cup V|} .
$$

To measure the Jaccard similarity between two fuzzy sets $A, B \in T 1(X)$ this is written as [1]

$$
s^{T 1}(A, B)=\frac{\sum_{i=1}^{n} \min \left(\mu_{A}\left(x_{i}\right), \mu_{B}\left(x_{i}\right)\right)}{\sum_{i=1}^{n} \max \left(\mu_{A}\left(x_{i}\right), \mu_{B}\left(x_{i}\right)\right)} .
$$

where $n$ is the total number of discretised points on the $x$-axis. This follows all four properties of a similarity measure.
The Jaccard measure $s^{T 1}$ has been extended to compare interval type-2 fuzzy sets $\tilde{A}, \tilde{B} \in I T 2(X)$ [4], [32] as

$$
\begin{aligned}
& s^{I T 2}(\tilde{A}, \tilde{B})= \\
& \quad \frac{\sum_{i=1}^{n} \min \left(\bar{\mu}_{\tilde{A}}\left(x_{i}\right), \bar{\mu}_{\tilde{B}}\left(x_{i}\right)\right)+\sum_{i=1}^{n} \min \left(\underline{\mu}_{\tilde{A}}\left(x_{i}\right), \underline{\mu}_{\tilde{B}}\left(x_{i}\right)\right)}{\sum_{i=1}^{n} \max \left(\bar{\mu}_{\tilde{A}}\left(x_{i}\right), \bar{\mu}_{\tilde{B}}\left(x_{i}\right)\right)+\sum_{i=1}^{n} \max \left(\underline{\mu}_{\tilde{A}}\left(x_{i}\right), \underline{\mu}_{\tilde{B}}\left(x_{i}\right)\right)} .
\end{aligned}
$$

This also follows all four properties of a similarity measure.

In recent years, a small handful of methods have been developed to measure the Jaccard ratio between two general type-2 fuzzy sets. The next three approaches all use the zSlices representation to measure the Jaccard similarity between fuzzy sets. Each method compares two zSlices using $s^{I T 2}$.

McCulloch et al. [6] used the interval type-2 Jaccard approach to compare fuzzy sets at each zLevel, aggregating and weighting the results according to the position of the zLevel. For $\tilde{A}, \tilde{B} \in G T 2(X)$, this is calculated as

$$
s_{m}^{G T 2}(\tilde{A}, \tilde{B})=\frac{\sum_{i \in p(\tilde{A}, \tilde{B})} z_{i} s^{I T 2}\left(\tilde{A}_{z_{i}}, \tilde{B}_{z_{i}}\right)}{\sum_{i \in p(\tilde{A}, \tilde{B})} z_{i}},
$$

where $p(\tilde{A}, \tilde{B})$ is the set created by the union of the zLevels used by $\tilde{A}$ and $\tilde{B}$ calculated as

$$
p(\tilde{A}, \tilde{B})=\tilde{A}_{Z} \cup \tilde{B}_{Z},
$$

where $\tilde{A}_{Z}$ and $\tilde{B}_{Z}$ are the set of zLevels in $\tilde{A}$ and $\tilde{B}$, respectively, as defined in (9).

Zhao et al. [7] proposed two new measures of similarity on type-2 fuzzy sets. One represents similarity as a fuzzy set and the other represents similarity as a crisp value as [7]

$$
s_{z h}^{G T 2}(A, B)=\frac{1}{\Delta+1} \sum_{i=0, \frac{1}{\Delta}, \frac{2}{\Delta} \cdots, \frac{\Delta-1}{\Delta}, 1} s^{I T 2}\left(\tilde{A}_{z_{i}}, \tilde{B}_{z_{i}}\right),
$$

where $\Delta+1$ is the number of zSlices representing $\tilde{A}$ and $\tilde{B}$. Note that, for consistency, the notations within (16) have been altered to match the zSlices notations. This approach follows all four properties of similarity. This differs from $s_{m}^{G T 2}(14)$ in that it does not weight the similarities of the zSlices by their zLevels and instead takes an unweighted average.

Note, the approach in $s_{z h}^{G T 2}$ [7] is also akin to the similarity measure proposed by Hamrawi and Coupland [33], which does not specify using the Jaccard measure.

Hao and Mendel [8] also developed a measure that represents the similarity between zSlices general type-2 fuzzy sets as a type-1 fuzzy set. This is expressed as

$$
s_{h}^{G T 2: F}(\tilde{A}, \tilde{B})=\bigcup_{\forall z_{i}} z_{i} / s^{I T 2}\left(\tilde{A}_{z_{i}}, \tilde{B}_{z_{i}}\right),
$$

where $z_{i} / s^{I T 2}\left(\tilde{A}_{z_{i}}, \tilde{B}_{z_{i}}\right)$ is not division but instead denotes the membership $z_{i}$ of the similarity resulting from $s^{I T 2}$; this is the same notation as used for the type- 1 fuzzy set representation in (2).

To demonstrate $s_{h}^{G T 2: F}$ (17), consider two zSlices general type-2 fuzzy sets $\tilde{A}$ and $\tilde{B}$, of which the lowest zSlices at $z_{1}$ are shown in Fig. 4; more details on the construction 


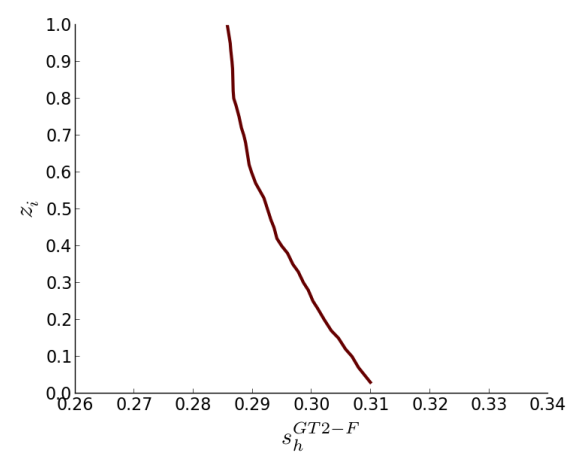

Fig. 1. The similarity $s_{h}^{G T 2: F}(\tilde{A}, \tilde{B})$ using the fuzzy sets $\tilde{A}$ and $\tilde{B}$ shown in Fig. 4.

of these fuzzy sets are given in Section IV. The similarity $s_{h}^{G T 2: F}(\tilde{A}, \tilde{B})(17)$ is shown in Fig. 1 .

The resulting fuzzy set from $s_{h}^{G T 2: F}$ (17) may be reduced to a crisp value by computing its centroid. This is calculated as

$$
s_{h}^{G T 2: C}(\tilde{A}, \tilde{B})=\frac{\sum_{i=1}^{I} z_{i} s_{h}^{G T 2: F}\left(\tilde{A}_{z_{i}}, \tilde{B}_{z_{i}}\right)}{\sum_{i=1}^{I} z_{i}} .
$$

This also follows all four properties of similarity. Note that when the fuzzy similarity $s_{h}^{G T 2: F}$ is reduced to a crisp value in $s_{h}^{G T 2: C}$, the result is the same as $s_{m}^{G T 2}$ (14).

This section has given an overview of similarity measures that compare fuzzy sets using the zSlices representation. The methods $s_{z h}^{G T 2}$ and $s_{h}^{G T 2: C}$ both assume that the fuzzy sets have normal secondary membership functions (i.e., $h_{z}=1$ ) and always compare the zSlice at $z_{i}=1.0$, therefore they cannot compare fuzzy sets that are non-normal. Additionally, $s_{m}^{G T 2}$ assumes that both fuzzy sets have identical secondary heights. Building upon these, the next section presents a new similarity measure that can compare fuzzy sets that may have both normal and non-normal secondary membership functions.

\section{A New Similarity Measure for General Type-2 FUZZY SETS}

This section expands upon the measures highlighted in the previous section and introduces a new method of measuring the similarity between general type-2 fuzzy sets where the given fuzzy sets may have non-normal secondary membership functions.

\section{A. Rationale}

When comparing two fuzzy sets $\tilde{A}, \tilde{B} \in G T 2(X)$ that may have non-normal secondary membership functions, two situations must be considered. These are:

1) How can we measure the similarity between fuzzy sets if $h_{z}(\tilde{A})<h_{z}(\tilde{B}) \leq 1$ ?

2) How can we measure the similarity between fuzzy sets if $h_{z}(\tilde{A})=h_{z}(\tilde{B})<1$ ?

where $h_{z}(\tilde{A})$ is defined in (10).

Fig. 2 gives an example of point 1 . This shows a threedimensional model of two fuzzy sets $\tilde{A}, \tilde{B} \in G T 2(X)$, where $\tilde{A}$ is represented by two zSlices at zLevels 0.25 , and 0.5 , and

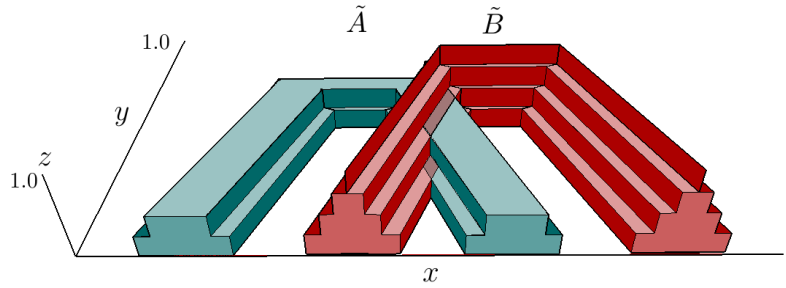

Fig. 2. Two fuzzy sets $\tilde{A}, \tilde{B} \in G T 2(X)$ where $\tilde{A}$ has 2 zSlices at zLevels 0.25 , and 0.5 , and $\tilde{B}$ is represented by four zSlices at zLevels $0.25,0.5,0.75$ and 1.0.

$\tilde{B}$ is represented by four zSlices at zLevels $0.25,0.5,0.75$ and 1.0 .

To compare these fuzzy sets, consider the property of overlapping (detailed in Section II-B). Using the Jaccard measure $s^{I T 2}$, for any given value of $x$ in $\tilde{A}, \tilde{B} \in I T 2(X)$, if $\bar{\mu}_{\tilde{A}}(x)>0$ and $\bar{\mu}_{\tilde{B}}(x)=0$ then the similarity at this given point will be 0 . In other words, if one fuzzy set has a nonzero membership at $x$ and the other has zero membership, then their similarity at $x$ is zero.

This point of view can also be considered in the secondary membership functions. If the primary membership value at $x$ is non-zero then the secondary membership will also be nonzero. Likewise, if the primary membership at $x$ is zero then the secondary membership must also be zero. Note that when comparing the zSlices of fuzzy sets, it is not just important that the secondary membership is non-zero, it must also have a non-empty zSlice at the given zLevel.

Therefore, when comparing fuzzy sets at a given zLevel $z_{i}$, if one fuzzy set has a zSlice at $z_{i}$ for $x$ and the other does not, then their similarity at $x$ is zero. More specifically, for $\tilde{A}, \tilde{B} \in G T 2(X)$ at $z_{i}$, if $h(\tilde{A})<z_{i}$ and $h(\tilde{B}) \geq z_{i}$ then $s^{I T 2}\left(\tilde{A}_{z_{i}}, \tilde{B}_{z_{i}}\right)=0$.

Next, regarding the second point, it is necessary to consider how we can compare two fuzzy sets that both have non-normal secondary heights. Fig. 3 illustrates this case, showing two fuzzy sets $\tilde{A}, \tilde{B} \in G T 2(X)$, both of which are represented by two zSlices at zLevels 0.25 and 0.5

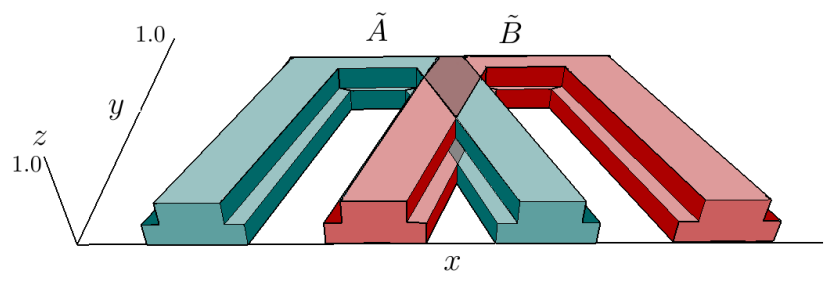

Fig. 3. Two fuzzy sets $\tilde{A}, \tilde{B} \in G T 2(X)$ where both $\tilde{A}$ and $\tilde{B}$ have 2 zSlices at zLevels 0.25 , and 0.5 .

To compare these fuzzy sets, consider the property of reflexivity. The method $s_{m}^{G T 2}$ (14) aggregates the similarities of zSlices by weighting the results by the given zLevel. Consider that, unlike the previous point, the similarity at $z_{i}$ where both $h(\tilde{A})<z_{i}$ and $h(\tilde{B})<z_{i}$ should not be 0 because this will break the property of reflexivity. For example, if two fuzzy sets $\tilde{A}, \tilde{B} \in G T 2(X)$ are identical and have a non-normal 
secondary height at $z_{h}$, then the similarity at $z_{i} \leq z_{h}$ would be 1 . If the result at $z_{i}>z_{h}$ is 0 then the value of similarity aggregated over all zSlices will be less than 1 . Considering this, fuzzy sets should not be compared at $z_{i}$ where $\tilde{A}_{z_{i}}=\emptyset$ and $\tilde{B}_{z_{i}}=\emptyset$.

Note that although one may consider the result should be 1 at such zLevels because the zSlices are both equal (i.e., they are both empty sets), this is not an ideal solution. This is because doing so would break the property of overlapping, causing two disjoint fuzzy sets with non-normal secondary membership functions to have a non-zero result.

\section{B. New Similarity Measure}

Considering the points discussed, the following similarity measure between two general type- 2 fuzzy sets is proposed.

Definition 14. The similarity between two fuzzy sets $\tilde{A}, \tilde{B} \in$ $G T 2(X)$ is calculated as

$$
s_{m 2}^{G T 2}(\tilde{A}, \tilde{B})=\frac{\sum_{i \in p(\tilde{A}, \tilde{B})} z_{i} s_{m 2}^{z I T 2}\left(\tilde{A}_{z_{i}}, \tilde{B}_{z_{i}}\right)}{\sum_{i \in p(\tilde{A}, \tilde{B})} z_{i}}
$$

where $p(\tilde{A}, \tilde{B})$ is given in (15), and the similarity between two zSlices $\tilde{A}_{z_{i}}, \tilde{B}_{z_{i}} \in z I T 2(X)$ is

$$
s_{m 2}^{z I T 2}\left(\tilde{A}_{z_{i}}, \tilde{B}_{z_{i}}\right)= \begin{cases}s^{I T 2}\left(\tilde{A}_{z_{i}}, \tilde{B}_{z_{i}}\right) & \tilde{A}_{z_{i}} \neq \emptyset \text { and } \tilde{B}_{z_{i}} \neq \emptyset \\ 0 & \tilde{A}_{z_{i}} \neq \emptyset \text { and } \tilde{B}_{z_{i}}=\emptyset \\ 0 & \tilde{A}_{z_{i}}=\emptyset \text { and } \tilde{B}_{z_{i}} \neq \emptyset\end{cases}
$$

Note that in $s_{m 2}^{z I T 2}(20)$, the case $\tilde{A}_{z_{i}}=\emptyset$ and $\tilde{B}_{z_{i}}=\emptyset$ is not included because such zLevels will not be used as set by $p(15)$. The maximum zLevel in $p(\tilde{A}, \tilde{B})$ will always be $\max \left\{h_{z}(\tilde{A}), h_{z}(\tilde{B})\right\}$, thus the fuzzy sets will never be compared where they both have an empty zSlice at the given zLevel. This is unlike the methods $s_{z h}^{G T 2}$ (16) and $s_{h}^{G T 2: C}$ (18) which both always measure up to $z_{i}=1$.

Note that the key difference between $s_{m 2}^{G T 2}$ (19) and $s_{m}^{G T 2}$ (14) is the introduction of measuring the similarity between fuzzy sets with different secondary heights. Additionally, although $s_{m}^{G T 2}$ can compare fuzzy sets with equal non-normal secondary heights, this was not explored within [6].

The proposed method (19) weights the similarity at each zLevel according to the position of the zLevel. This is because, intuitively, the more certainty there is in the membership value of the given sets, the more certainty there must be in the similarity between these sets. Given this, it makes sense to weight the similarity between zSlices to reflect how certain we are of that similarity.

Theorem 1. $s_{m 2}^{G T 2}$ has the property of reflexivity.

Proof: For $\tilde{A}, \tilde{B} \in G T 2(X)$, if $\tilde{A}=\tilde{B}$ then $h_{z}(\tilde{A})=$ $h_{z}(\tilde{B})$ so both fuzzy sets can be measured up to the same zLevel. It then follows that $s^{z I T 2}\left(\tilde{A}_{z_{i}} \tilde{B}_{z_{i}}\right)=1 \forall z_{i} \in p(\tilde{A}, \tilde{B})$. Thus, $s_{m 2}^{G T 2}$ has the property of reflexivity.

Theorem 2. $s_{m 2}^{G T 2}$ has the property of symmetry.
Proof: The function $s_{m 2}^{G T 2}$ does not affect the ordering of the fuzzy sets measured, thus it follows the rule of symmetry.

Theorem 3. $s_{m 2}^{G T 2}$ has the property of overlapping.

Proof: For $\tilde{A}, \tilde{B} \in G T 2(X)$, if $\tilde{A}$ and $\tilde{B}$ are disjoint then they are disjoint at each zLevel, therefore $s_{m 2}^{z I T 2}\left(\tilde{A}_{z_{i}} \tilde{B}_{z_{i}}\right)=$ $0 \forall z_{i} \in p(\tilde{A}, \tilde{B})$. Thus, $s_{m 2}^{G T 2}$ has the property of overlapping.

Theorem 4. $s_{m 2}^{G T 2}$ has the property of transitivity.

Proof: When fuzzy sets have equal heights in the secondary membership functions:

For $\tilde{A}, \tilde{B}, \tilde{C} \in G T 2(X)$ where $\tilde{A} \subseteq \tilde{B} \subseteq \tilde{C}$, for each non-empty zSlice $z_{i}, \tilde{A}_{z_{i}} \subseteq \tilde{B}_{z_{i}} \subseteq \tilde{C}_{z_{i}}$ and therefore $s^{I T 2}\left(\tilde{A}_{z_{i}}, \tilde{B}_{z_{i}}\right) \geq s^{I T 2}\left(\tilde{A}_{z_{i}}, \tilde{C}_{z_{i}}\right)$ [4]. Thus, it is clear that when $h_{z}(\tilde{A})=h_{z}(\tilde{B})=h_{z}(\tilde{C})$, the property of transitivity holds.

When fuzzy sets have non-equal heights in secondary membership functions:

Let $z_{\tilde{A}}, z_{\tilde{B}}$ and $z_{\tilde{C}}$ denote the heights of the fuzzy sets; i.e., $z_{\tilde{A}}=h_{z}(\tilde{A}), z_{\tilde{B}}=h_{z}(\tilde{B})$ and $z_{\tilde{C}}=h_{z}(\tilde{C})$. If $\tilde{A} \subseteq \tilde{B} \subseteq \tilde{C}$ then $z_{\tilde{A}} \leq z_{\tilde{B}} \leq z_{\tilde{C}}$ and for each non-empty zSlice $z_{i}$, $\tilde{A}_{z_{i}} \subseteq \tilde{B}_{z_{i}} \subseteq \tilde{C}_{z_{i}}$.

At $z_{i} \in\left(0, z_{\tilde{A}}\right], s_{m 2}^{z I T 2}\left(\tilde{A}_{z_{i}}, \tilde{B}_{z_{i}}\right) \geq s_{m 2}^{z I T 2}\left(\tilde{A}_{z_{i}}, \tilde{C}_{z_{i}}\right)$

At $z_{i} \in\left(z_{\tilde{A}}, z_{\tilde{B}}\right], s_{m 2}^{z I T 2}\left(\tilde{A}_{z_{i}}, \tilde{B}_{z_{i}}\right)=0$ and $s_{m 2}^{z I T 2}\left(\tilde{A}_{z_{i}}, \tilde{C}_{z_{i}}\right)=$ 0 , thus $s_{m 2}^{z I T 2}\left(\tilde{A}_{z_{i}}, \tilde{B}_{z_{i}}\right) \geq s_{m 2}^{z I T 2}\left(\tilde{A}_{z_{i}}, \tilde{C}_{z_{i}}\right)$.

At $z_{i} \in\left(z_{\tilde{B}}, z_{\tilde{C}}\right], s_{m 2}^{z I T 2}\left(\tilde{A}_{z_{i}}, \tilde{B}_{z_{i}}\right)$ is not measured because $z_{i}$ exceeds the heights $z_{\tilde{A}}$ and $z_{\tilde{B}}$, and $s_{m 2}^{z I T 2}\left(\tilde{A}_{z_{i}}, \tilde{C}_{z_{i}}\right)=$ 0 . Thus, the similarity at $z_{i}$ decreases the value of $s_{m 2}^{G T 2}(\tilde{A}, \tilde{C})$, whilst $s_{m 2}^{G T 2}(\tilde{A}, \tilde{B})$ remains the same. Therefore, $s_{m 2}^{G T 2}(\tilde{A}, \tilde{B}) \geq s_{m 2}^{G T 2}(\tilde{A}, \tilde{C})$.

This section has developed a new method of comparing zSlices-based general type-2 fuzzy sets that may have nonnormal secondary membership functions. The next section demonstrates this measure on a variety of fuzzy sets compared with other approaches within the literature.

\section{DEMONSTRATIONS}

This section demonstrates the proposed similarity measure. First, a demonstration is given where all fuzzy sets have normal secondary membership functions, and the results are compared against existing methods introduced in Section II-B. After this, the proposed method is demonstrated on fuzzy set pairs where one fuzzy set is normal $\left(h_{z}=1\right)$ and the other is non-normal $\left(h_{z}<1\right)$, and pairs in which both fuzzy sets are non-normal. Each fuzzy set is constructed using simple triangular membership functions so that the properties proved in the previous section can easily be seen through the results. However, note that the methodology is applicable to fuzzy sets with any membership function shape, including non-normal and non-convex.

First, the method of constructing the fuzzy sets used within these demonstrations is discussed. 


\section{A. Generating Sample General Type-2 Fuzzy Sets}

Let $\tilde{A} \in G T 2(X)$ be a fuzzy set with triangular upper and lower membership functions for each zSlice. The footprint of uncertainty (FOU) of the lowest zSlice $\left(z_{1}\right)$ of $\tilde{A}$ is defined by the upper and lower membership functions as

$$
\begin{aligned}
& \overline{\operatorname{FOU}\left(\tilde{A}_{z_{1}}\right)}=\operatorname{trimf}(x,[\bar{a}, \bar{b}, \bar{c} ; \bar{w}]) \\
& \underline{\operatorname{FOU}\left(\tilde{A}_{z_{1}}\right)}=\operatorname{trimf}(x,[\underline{a}, \underline{b}, \underline{c} ; \underline{w}]) .
\end{aligned}
$$

The secondary membership functions of $\tilde{A}$ have maximum membership (i.e., $\mu_{\tilde{A}}(x, u)=1$ ) at the centre of the FOU and the membership decreases linearly towards the edge of the FOU. Fig. 5 shows an example of such secondary membership functions using five zSlices.

For any given zLevel $z_{i}$ where $I$ is the total number of zLevels, the upper membership function of $\tilde{A}_{z_{i}}$ is

$$
\overline{\operatorname{FOU}\left(\tilde{A}_{z_{i}}\right)}=\operatorname{trimf}\left(x,\left[\overline{a_{z_{i}}}, \overline{b_{z_{i}}}, \overline{c_{z_{i}}} ; \overline{w_{z_{i}}}\right]\right)
$$

where

$$
\begin{aligned}
& \overline{a_{z_{i}}}=\bar{a}+\left((\underline{a}-\bar{a})\left(\frac{z_{i}}{I-1}\right)\right) \\
& \overline{b_{z_{i}}}=\bar{b}+\left((\underline{b}-\bar{b})\left(\frac{z_{i}}{I-1}\right)\right) \\
& \ldots
\end{aligned}
$$

and the lower membership function of $\tilde{A}_{z_{i}}$ is

$$
\underline{\operatorname{FOU}\left(\tilde{A}_{z_{i}}\right)}=\operatorname{trimf}\left(x,\left[\underline{a_{z_{i}}}, \underline{b_{z_{i}}}, \underline{c_{z_{i}}} ; \underline{w_{z_{i}}}\right]\right)
$$

where

$$
\begin{aligned}
& \underline{a_{z_{i}}}=\underline{a}-\left((\underline{a}-\bar{a})\left(\frac{z_{i}}{I-1}\right)\right) \\
& \underline{b_{z_{i}}}=\underline{b}-\left((\underline{b}-\bar{b})\left(\frac{z_{i}}{I-1}\right)\right)
\end{aligned}
$$

The demonstrations within this section use five fuzzy sets $\tilde{A}, \tilde{B}, \tilde{C}, \tilde{D}, \tilde{E} \in G T 2(X)$ where each zSlice is calculated as described above. Table I lists the upper and lower membership functions of each fuzzy set at $z_{1}$.

TABLE I

THE LOWER AND UPPER MEMBERSHIP FUNCTIONS OF FIVE FUZZY SETS AT THE ZLEVEL $z_{1}$, GIVEN AS TRIANGULAR MEMBERSHIP FUNCTIONS DEFINED BY THE POINTS $[a, b, c ; w]$.

\begin{tabular}{ccc}
\hline Fuzzy Set & $\overline{F O U}$ & $\underline{F O U}$ \\
\hline$\tilde{A}_{z_{1}}$ & {$[1,3,5 ; 1.0]$} & {$[2,3,4 ; 0.8]$} \\
$\tilde{B}_{z_{1}}$ & {$[2,4,6 ; 1.0]$} & {$[3,4,5 ; 0.8]$} \\
$\tilde{C}_{z_{1}}$ & {$[3,5,7 ; 1.0]$} & {$[4,5,6 ; 0.8]$} \\
$\tilde{D}_{z_{1}}$ & {$[4,6,8 ; 1.0]$} & {$[5,6,7 ; 0.8]$} \\
$\tilde{E}_{z_{1}}$ & {$[5,7,9 ; 1.0]$} & {$[6,7,8 ; 0.8]$} \\
\hline
\end{tabular}

Fig. 4 shows the FOU of each fuzzy set for the zSlice at $z_{1}$ and Fig. 5 shows a three-dimensional model of $\tilde{A}$ represented by five zSlices.

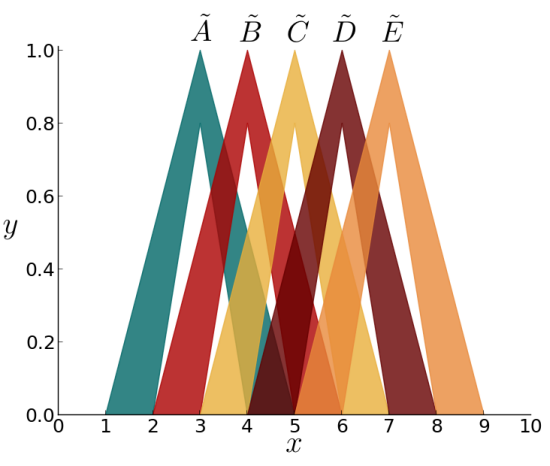

Fig. 4. The footprint of uncertainty of five fuzzy sets $\tilde{A}, \tilde{B}, \tilde{C}, \tilde{D}, \tilde{E} \in$ $G T 2(X)$ at the zSlice $\tilde{Z}_{1}$.

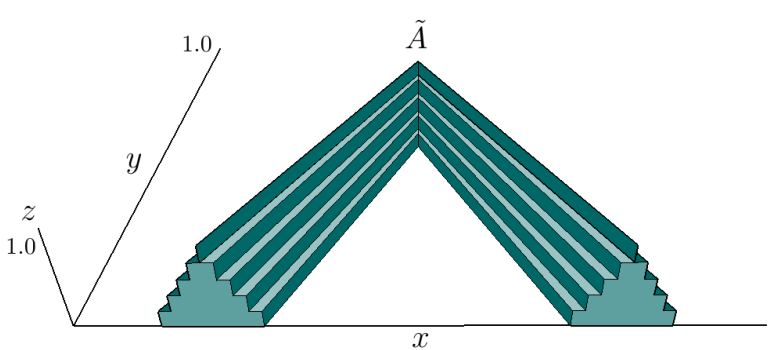

Fig. 5. The fuzzy set $\tilde{A}$ from Fig. 4 represented by five zSlices.

Note that this method assures that $\operatorname{FOU}\left(\tilde{A}_{z_{i}}\right)(x)>$ $\operatorname{FOU}\left(\tilde{A}_{z_{j}}\right)(x)$ and $\overline{F O U\left(\tilde{A}_{z_{i}}\right)}(x)<\overline{\operatorname{FOU}\left(\tilde{A}_{z_{j}}\right)}(x)$ where $z_{i}>z_{j}$. In other words, a zSlice at $z_{i}$ has an FOU that is within the FOU of the zSlice at $z_{j}$ where $z_{i}>z_{j}$.

\section{B. Similarity Between Fuzzy Sets with Normal Secondary Membership Functions}

This demonstration compares the fuzzy sets described in the previous section using the proposed method $s_{m 2}^{G T 2}$ (19), along with $s_{m}^{G T 2}$ (14), $s_{z h}^{G T 2}$ (16) and $s_{h}^{G T 2}$ (18). Each fuzzy set has normal secondary membership functions and is represented by 20 zSlices (at zLevels $z=\{0.05,0.1, \ldots, 0.95,1.0\}$ ). Note that for each demonstration, the universe of discourse $X$ is bounded by the interval $[0,10]$ and $n=101$ (i.e., $X$ is discretised into 101 equidistant points).

Table II shows the results of each method when comparing different pairs of fuzzy sets in Fig. 4. It is clear from these results that when each fuzzy set has a secondary height of 1 , the proposed method $s_{m 2}^{G T 2}$, McCulloch et al.'s approach $s_{m}^{G T 2}$ and Hao and Mendel's method $s_{h}^{G T 2}$ all produce the same results. Zhao et al. $s_{z h}^{G T 2}$ produce different results; however, the values are close to the other methods. Note, also, that each method produces expected values and follows all four properties of similarity.

\section{Similarity Between Fuzzy Sets with Normal and Non- Normal Secondary Membership Functions}

Next, a demonstration of $s_{m 2}^{G T 2}$ is given where all fuzzy sets except $\tilde{A}$ have non-normal secondary membership functions. In the fuzzy sets $\tilde{B}, \tilde{C}, \tilde{D}$ and $\tilde{E}$, all zSlices above $z_{i}=0.75$ 
TABLE II

RESULTS OF COMPARING THE FUZZY SETS IN FIG. 4 WHERE EACH FUZZY SET HAS A NORMAL SECONDARY MEMBERSHIP FUNCTION.

\begin{tabular}{lcccccc}
\hline Method & $s(\tilde{A}, \tilde{A})$ & $s(\tilde{A}, \tilde{B})$ & $s(\tilde{B}, \tilde{A})$ & $s(\tilde{A}, \tilde{C})$ & $s(\tilde{A}, \tilde{D})$ & $s(\tilde{A}, \tilde{E})$ \\
\hline$s_{m 2}^{G T 2}(19)$ & 1.0 & 0.2902 & 0.2902 & 0.0658 & 0.0044 & 0.0 \\
$s_{m}^{G T 2}(14)$ & 1.0 & 0.2902 & 0.2902 & 0.0658 & 0.0044 & 0.0 \\
$s_{z h}^{G T 2}(16)$ & 1.0 & 0.2943 & 0.2943 & 0.0723 & 0.0083 & 0.0 \\
$s_{h}^{G T 2: C}(18)$ & 1.0 & 0.2902 & 0.2902 & 0.0658 & 0.0044 & 0.0 \\
\hline
\end{tabular}

have been removed and the fuzzy sets are now represented by 15 zSlices (at zLevels $z=\{0.05,0.1, \ldots, 0.7,0.75\}$ ).

Additionally, $\tilde{A}^{\prime}$ and $\tilde{A}^{\prime \prime}$ are introduced into this demonstration. These are the same as $\tilde{A}$ but in $\tilde{A}^{\prime}$ the zSlices above $z_{i}=0.75$ have also been removed, thus $\tilde{A}^{\prime}$ has also been reduced to $15 \mathrm{zSlices}$ where the maximum zSlice is at $z_{i}=0.75$. However, in $\tilde{A}^{\prime \prime}$ the zSlices below $z_{i}=0.25$ have been removed, thus $\tilde{A}^{\prime \prime}$ has been reduced to $15 \mathrm{zSlices}$ at zLevels $z=\{0.25,0.3, \ldots, 0.98,1.0\}$. To help visualise these fuzzy sets, Fig. 6 shows the vertical slices of $\tilde{A}, \tilde{A}^{\prime}$ and $\tilde{A}^{\prime \prime}$ at $x=3.4$. Note that $\tilde{A}^{\prime}$ has a non-normal secondary membership function, and the FOU of $\tilde{A}^{\prime \prime}$ at $z \leq 0.25$ is the same as the $\tilde{A}$ and $\tilde{A}^{\prime}$ at $z=0.25$.

Table III shows the results of comparing these fuzzy sets. Note that only the results of the proposed method $s_{m 2}^{G T 2}$ are shown as $s_{m}^{G T 2}, s_{z h}^{G T 2}$ and $s_{h}^{G T 2: C}$ cannot be used to compare fuzzy sets with non-normal secondary membership functions.

As expected, the similarity between $\tilde{A}$ and $\tilde{A}^{\prime}$ is lower than when $\tilde{A}$ is compared with itself (shown in Table II). However, since $75 \%$ of the $\tilde{A}$ and $\tilde{A}^{\prime}$ are identical, this similarity is still high. Note that the similarity is less than 0.75 because lower weights are given to the values of similarity at lower zLevels, thus the result of $s_{m 2}^{G T 2}$ is less than 0.75 .

In contrast, the similarity between $\tilde{A}$ and $\tilde{A}^{\prime \prime}$ is higher. This is because the zSlices with higher degrees of secondary

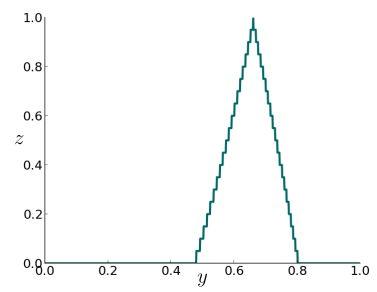

(a)

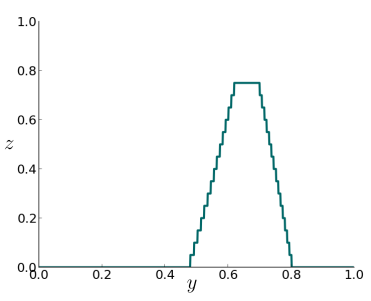

(b)

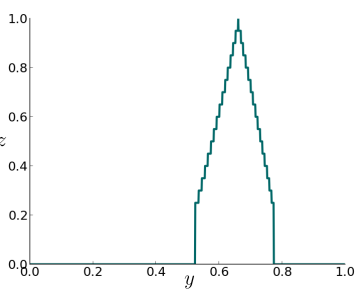

(c)
Fig. 6. Vertical slices at $x=3.4$ of (a) $\tilde{A}$ (b) $\tilde{A}^{\prime}$ and (c) $\tilde{A}^{\prime \prime}$.
TABLE III

RESULTS OF COMPARING THE FUZZY SETS IN FIG. 4 USING $s_{m 2}^{G T 2}(19)$, WHERE $\tilde{A}$ AND $\tilde{A}^{\prime \prime}$ HAVE NORMAL SECONDARY MEMBERSHIP FUNCTIONS, AND $\tilde{A}^{\prime}, \tilde{B}, \tilde{C}, \tilde{D}$ AND $\tilde{E}$ ARE NON-NORMAL.

\begin{tabular}{ccccccc}
\hline$s\left(\tilde{A}, \tilde{A}^{\prime}\right)$ & $s\left(\tilde{A}, \tilde{A}^{\prime \prime}\right)$ & $s(\tilde{A}, \tilde{B})$ & $s(\tilde{B}, \tilde{A})$ & $s(\tilde{A}, \tilde{C})$ & $s(\tilde{A}, \tilde{D})$ & $s(\tilde{A}, \tilde{E})$ \\
\hline 0.5714 & 0.9979 & 0.1675 & 0.1675 & 0.0402 & 0.0042 & 0.0 \\
\hline
\end{tabular}

membership are identical and it is only at low degrees of membership (where $z_{i}<0.25$ ) that the fuzzy sets are different. As a result of weighting the similarity by the secondary membership values, the result of $\tilde{A}$ and $\tilde{A}^{\prime \prime}$ is close to 1 .

For all other pairs of fuzzy sets, the similarity is lower than if all secondary membership functions are at 1 (as demonstrated in Table II) because there is now no similarity at $z_{i}$ where $z_{i}>0.75$. As a result, the value of similarity at these zLevels is 0 and so the overall result (aggregating all zLevels) is reduced. The same effect can be observed on type-1 fuzzy sets with normal and non-normal membership functions.

Note that the results are as expected and demonstrate the properties of similarity.

\section{Similarity Between Fuzzy Sets with Non-Normal Secondary Membership Functions}

Next, a demonstration of $s_{m 2}^{G T 2}$ is given where all of the fuzzy sets have non-normal secondary membership functions. The zSlices above $z_{i}=0.75$ have been removed and all five fuzzy sets are now represented by 15 zSlices (at zLevels $z=$ $\{0.05,0.1, \ldots, 0.7,0.75\})$.

Table IV shows the results of comparing these fuzzy sets. For each pair, the similarity is higher than the previous demonstration given in Table III because each fuzzy set now has an equal secondary height, and therefore the zLevels above $z_{i}=0.75$ are not compared. This is unlike the previous demonstration in which the resulting similarity at those zLevels was 0 . It is expected that the results should be higher than in Table III because the fuzzy sets are now more similar; i.e., they all have the same secondary height.

The results are different compared to when the fuzzy sets all have normal secondary membership functions (shown in Table II) because the fuzzy sets themselves are different at $z_{i}>0.75$ even though they have the same FOU.

Note that the results are as expected and demonstrate the four properties of similarity.

These demonstrations show that the proposed method is effective at comparing zSlices based fuzzy sets with normal or non-normal secondary membership functions, and the results

TABLE IV

RESULTS OF COMPARING THE FUZZY SETS IN FIG. 4 USING $s_{m 2}^{G T 2}$ (19), WHERE EACH FUZZY SET HAS A NON-NORMAL SECONDARY MEMBERSHIP FUNCTION.

\begin{tabular}{cccccc}
\hline$s(\tilde{A}, \tilde{A})$ & $s(\tilde{A}, \tilde{B})$ & $s(\tilde{B}, \tilde{A})$ & $s(\tilde{A}, \tilde{C})$ & $s(\tilde{A}, \tilde{D})$ & $s(\tilde{A}, \tilde{E})$ \\
\hline 1.0 & 0.2932 & 0.2932 & 0.0704 & 0.0074 & 0.0 \\
\hline
\end{tabular}


are consistent with other methods that cannot compare such fuzzy sets.

\section{CONCLUSIONS}

This paper presents a novel method of measuring the similarity between two zSlices/ $\alpha$-plane based type- 2 fuzzy sets that may have non-normal secondary membership functions. Though several methods of calculating the similarity between type-2 fuzzy sets have been developed in the literature, there are none that can compare such sets if the secondary membership functions of the fuzzy sets are non-normal. Such fuzzy sets may arise when, for example, modelling agreement if there is no unanimity between individuals or, in some contexts, it may make sense to describe a term as not ever being completely certain [20].

This paper develops a new measure by building on existing techniques to enable the systematic comparison of general type-2 fuzzy sets with both normal and non-normal secondary membership functions. It also provides proofs that the proposed method has all of the properties typically desired in a similarity measure. Examples are given to compare fuzzy sets with normal and non-normal secondary membership functions. These examples illustrate that the results are as expected and are consistent with other methods in the literature. The results also demonstrate the effects of the properties of the similarity measure when comparing fuzzy sets; i.e., when comparing identical or disjoint fuzzy sets. The proposed method may be used in type-2 fuzzy set applications where a measure with all four properties of a similarity measure are required.

\section{REFERENCES}

[1] R. Zwick, E. Carlstein, and D. V. Budescu, "Measures of similarity among fuzzy concepts: A comparative analysis," International Journal of Approximate Reasoning, vol. 1, no. 2, pp. 221-242, 1987.

[2] X. Wang, B. De Baets, and E. Kerre, "A comparative study of similarity measures," Fuzzy sets and systems, vol. 73, no. 2, pp. 259-268, 1995.

[3] V. V. Cross and T. A. Sudkamp, Similarity and compatibility in fuzzy set theory: assessment and applications. Springer Science \& Business Media, 2002, vol. 93.

[4] D. Wu and J. M. Mendel, "A comparative study of ranking methods, similarity measures and uncertainty measures for interval type-2 fuzzy sets," Information Sciences, vol. 179, no. 8, pp. 1169-1192, 2009.

[5] G. Zheng, J. Wang, W. Zhou, and Y. Zhang, "A similarity measure between interval type-2 fuzzy sets," in Mechatronics and Automation (ICMA), 2010 International Conference on, Aug. 2010, pp. 191-195.

[6] J. McCulloch, C. Wagner, and U. Aickelin, "Extending similarity measures of interval type-2 fuzzy sets to general type-2 fuzzy sets," in Fuzzy Systems (FUZZ), 2013 IEEE International Conference on, 2013, pp. 1-8.

[7] T. Zhao, J. Xiao, Y. Li, and X. Deng, "A new approach to similarity and inclusion measures between general type-2 fuzzy sets," Soft Computing, vol. 18, no. 4, pp. 809-823, 2014.

[8] M. Hao and J. M. Mendel, "Similarity measures for general type-2 fuzzy sets based on the $\alpha$-plane representation," Information Sciences, vol. 277, no. 0, pp. 197-215, 2014.

[9] H. Mitchell, "Pattern recognition using type-II fuzzy sets," Information Sciences, vol. 170, no. 2-4, pp. 409-418, 2005.

[10] M.-S. Yang and D.-C. Lin, "On similarity and inclusion measures between type-2 fuzzy sets with an application to clustering," Computers \& Mathematics with Applications, vol. 57, no. 6, pp. 896-907, 2009.

[11] C. Wagner, S. Miller, and J. Garibaldi, "Similarity based applications for data-driven concept and word models based on type-1 and type-2 fuzzy sets," in Fuzzy Systems (FUZZ), 2013 IEEE International Conference on, 2013, pp. 1-9.
[12] B. Bouchon-Meunier, M. Rifqi, and M.-J. Lesot, "Similarities in Fuzzy Data Mining: From a Cognitive View to Real-World Applications," in Computational Intelligence: Research Frontiers, ser. Lecture Notes in Computer Science, J. Zurada, G. Yen, and J. Wang, Eds. Springer Berlin Heidelberg, 2008, vol. 5050, pp. 349-367.

[13] C.-M. Hwang, M.-S. Yang, W.-L. Hung, and E. S. Lee, "Similarity, inclusion and entropy measures between type-2 fuzzy sets based on the Sugeno integral," Mathematical and Computer Modelling, vol. 53, no. 9-10, pp. 1788-1797, 2011.

[14] D. Li, T. Li, and T. Zhao, "A New Clustering Method Based On Type-2 Fuzzy Similarity and Inclusion Measures," Journal of Computers, vol. 9, no. 11 , pp. 2559-2569, 2014

[15] C. Wagner, S. Miller, J. Garibaldi, D. Anderson, and T. Havens, "From Interval-Valued Data to General Type-2 Fuzzy Sets," IEEE Trans. Fuzzy Syst., vol. 23, no. 2, pp. 248-269, Apr. 2015.

[16] F. Doctor, C.-H. Syue, Y.-X. Liu, J.-S. Shieh, and R. Iqbal, "Type2 Fuzzy Sets Applied to Multivariable Self-Organizing Fuzzy Logic Controllers for Regulating Anesthesia," Applied Soft Computing, 2015.

[17] O. Linda and M. Manic, "General Type-2 Fuzzy C-Means Algorithm for Uncertain Fuzzy Clustering," IEEE Trans. Fuzzy Syst., vol. 20, no. 5, pp. 883-897, Oct. 2012.

[18] L. Livi, H. Tahayori, A. Sadeghian, and A. Rizzi, "Aggregating alphaplanes for Type-2 fuzzy set matching," in IFSA World Congress and NAFIPS Annual Meeting (IFSA/NAFIPS), 2013 Joint, 2013, pp. 860865.

[19] A. D. Torshizi and M. H. F. Zarandi, "Alpha-plane based automatic general type-2 fuzzy clustering based on simulated annealing metaheuristic algorithm for analyzing gene expression data," Computers in Biology and Medicine, vol. 64, pp. 347-359, 2015.

[20] J. Garibaldi and R. John, "Choosing membership functions of linguistic terms," in Fuzzy Systems, 2003. FUZZ '03. The 12th IEEE International Conference on, vol. 1, 2003, pp. 578-583.

[21] J. Mendel and R. John, "Type-2 fuzzy sets made simple," IEEE Trans. Fuzzy Syst., vol. 10, no. 2, pp. 117-127, Apr. 2002.

[22] J. Mendel, R. John, and F. Liu, "Interval Type-2 Fuzzy Logic Systems Made Simple," IEEE Trans. Fuzzy Syst., vol. 14, no. 6, pp. 808-821, Dec. 2006.

[23] C. Wagner and H. Hagras, "Toward General Type-2 Fuzzy Logic Systems Based on zSlices," IEEE Trans. Fuzzy Syst., vol. 18, no. 4, pp. 637-660, Aug. 2010.

[24] J. Mendel and F. Liu, "On new quasi-type-2 fuzzy logic systems," in Fuzzy Systems, 2008. FUZZ-IEEE 2008. (IEEE World Congress on Computational Intelligence). IEEE International Conference on, 2008, pp. 354-360.

[25] J. Mendel, F. Liu, and D. Zhai, " $\alpha$-Plane Representation for Type-2 Fuzzy Sets: Theory and Applications," IEEE Trans. Fuzzy Syst., vol. 17, no. 5, pp. 1189-1207, Oct. 2009.

[26] L. Zadeh, "Fuzzy sets," Information and Control, vol. 8, no. 3, pp. 338353, 1965.

[27] W. Zeng and Y. Shi, "Note on Interval-Valued Fuzzy Set," in Fuzzy Systems and Knowledge Discovery, ser. Lecture Notes in Computer Science, L. Wang and Y. Jin, Eds. Springer Berlin Heidelberg, 2005, vol. 3613, pp. 20-25.

[28] A. Tversky, "Features of Similarity," Psychological Review, vol. 84, no. 4, pp. 327-352, 1977.

[29] M. De Cock and E. Kerre, "On (un)suitable fuzzy relations to model approximate equality," Fuzzy Sets and Systems, vol. 133, no. 2, pp. 137$153,2003$.

[30] F. Klawonn, "Should fuzzy equality and similarity satisfy transitivity? Comments on the paper by M. De Cock and E. Kerre," Fuzzy Sets and Systems, vol. 133, no. 2, pp. 175-180, 2003.

[31] P. Jaccard, "The distribution of the flora in the alpine zone. 1," New phytologist, vol. 11 , no. 2, pp. 37-50, 1912.

[32] H. T. Nguyen and V. Kreinovich, "Computing Degrees of Subsethood and Similarity for Interval-Valued Fuzzy Sets: Fast Algorithms," Proceedings of the 9th International Conference on Intelligent Technologies InTec'08, pp. 47-55, 2008.

[33] H. Hamrawi and S. Coupland, "Measures of uncertainty for type-2 fuzzy sets," in Computational Intelligence (UKCI), 2010 UK Workshop on, 2010, pp. 1-6. 